Max-Planck-Institut für demografische Forschung Max Planck Institute for Demographic Research Konrad-Zuse-Strasse 1 - D-18057 Rostock - GERMANY

Tel +49 (0) 3812081 - 0; Fax +49 (0) 3812081 - 202;

http://www.demogr.mpg.de

MPIDR WORKING PAPER WP 2004-006

FEBRUARY 2004

\title{
Erwerbsstatus und \\ Familienentwicklung in Schweden aus paarbezogener Perspektive
}

\author{
Gunnar Andersson (andersson@ demogr.mpg.de) \\ Ann-Zofie Duvander (ann-zofie.duvander@rfv.sfa.se) \\ Karsten Hank (hank@mea.uni-mannheim.de)
}

This working paper has been approved for release by: Jan M. Hoem (hoem@ demogr.mpg.de)

Head of the Laboratory of Contemporary European Fertility and Family Dynamics.

() Copyright is held by the authors.

Working papers of the Max Planck Institute for Demographic Research receive only limited review.

Views or opinions expressed in working papers are attributable to the authors and do not necessarily reflect those of the Institute. 


\title{
Erwerbsstatus und Familienentwicklung in Schweden aus paarbezogener Perspektive
}

\author{
Gunnar Andersson ${ }^{a}$, Ann-Zofie Duvander ${ }^{b}$, und Karsten Hank ${ }^{c}$
}

Zusammenfassung: Der Beitrag untersucht die Frage nach der Bedeutung von Erwerbsmerkmalen schwedischer Männer und Frauen für den Prozess der Familienentwicklung in Schweden aus paarbezogener Perspektive. Auf der Grundlage von Registerdaten der Jahre 1981 bis 1999 schätzen wir ereignisanalytische Modelle für die Geburt zweiter und dritter Kinder. Es zeigt sich, dass Einkommen und Erwerbstätigkeit beider Partner weitgehend unabhängig voneinander auf die Wahrscheinlichkeit wirken, ein weiteres Kind zu bekommen. Die Richtung der bei Männern und Frauen etwa gleich starken Effekte weist (im Gegensatz etwa zu Annahmen der ,Neuen Haushaltsökonomie') auf einen grundsätzlich positiven Zusammenhang zwischen unseren Erwerbsvariablen und der Familienentwicklung hin. Der Befund, dass ein traditioneller Familienkontext Geburten höherer Ordnung in Schweden eher blockiert, weist auf ein vergleichsweise hohes Maß an sozialer Gleichheit zwischen den Geschlechtern hin.

\begin{abstract}
This paper investigates the role of men's and women's labor-market attachment in the process of family building in Sweden, taking the perspective of couples. Using register data for the years 1981 to 1999 we estimate event-history models for second and third births. It is shown that income and employment of both partners have a largely independent effect on the propensity to have another child. The effect is of similar size for men and women and points to a generally positive relationship between our employment variables and continued childbearing (which is contrary to assumptions suggested by the 'New Home Economics', for example). The finding that a traditional family context tends to hamper higher parity births in Sweden points to a relatively high degree of gender equity in that society.
\end{abstract}

\footnotetext{
a Max-Planck-Institut für demografische Forschung, Rostock (andersson@demogr.mpg.de).

${ }^{\mathrm{b}}$ National Social Insurance Board, Department of Research Analysis and Statistics, Stockholm (ann-zofie.duvander@rfv.sfa.se).

c Mannheimer Forschungsinstitut Ökonomie und demographischer Wandel, Universität Mannheim(hank@mea.uni-mannheim.de).
} 


\section{Einleitung}

Der individuelle Lebensverlauf besteht aus einer Vielzahl paralleler, bzw. interdependenter Prozesse. Entsprechend werden Fertilitätsentscheidungen nicht unabhängig von Ereignissen in anderen relevanten Lebensbereichen gefällt (z.B. Baizán et al. 2002; Blossfeld et al. 1996). So gehört es mittlerweile fast schon zum Alltagswissen, dass vor allem Frauen mit langen Ausbildungszeiten und hohen Bildungsabschlüssen die Familiengründung häufig aufschieben oder sogar ganz auf Kinder verzichten (z.B. Blossfeld und Huinink 1989; Kravdal 2001). Die Tatsache, dass Fertilitätsentscheidungen nicht monadisch getroffen werden, sondern als Interaktion zwischen (mindestens) zwei Individuen in einer Partnerschaft, hat bislang hingegen verhältnismäßig wenig Beachtung in der empirischen Literatur gefunden (vgl. Corijn et al. 1996; Klein 2003).

Eine der Ursachen hierfür dürfte die hohe Komplexität sein, die die explizite Modellierung partnerschaftlicher Entscheidungs- und Verhandlungsprozesse mit sich bringt (vgl. Kohlmann und Kopp 1997; Thomson 1990). Hinzu kommt, dass kaum Datensätze vorliegen, die in angemessener Weise Merkmale beider Partner beinhalten. Zwar gibt es inzwischen einige Datenquellen, die auch Informationen über Paare enthalten (z.B. das Sozio-oekonomische Panel oder der Familiensurvey); exakte Fertilitätsbiographien von Männern sind jedoch nach wie vor selten (vgl. Hank und Tölke 2004).

Eine Ausnahme in dieser Hinsicht stellt das schwedische Bevölkerungsregister dar, in dem - unabhängig vom Geschlecht - für alle Personen verlässliche Informationen über die Geburt von Kindern erfasst werden. Im vorliegenden Beitrag nutzen wir diese Registerdaten, um den Prozess der Familienentwicklung in Schweden 
während der 1980er und 1990er Jahre aus paarbezogener Perspektive zu analysieren (siehe auch Thomson und Hoem 1998). ${ }^{1}$ Im Vordergrund steht dabei die Frage nach der individuellen und der gemeinsamen Bedeutung von Erwerbsmerkmalen des Mannes und der Frau für die Wahrscheinlichkeit, dass ein (Eltern-)Paar ein zweites oder drittes Kind bekommt. Zwar existiert bereits eine umfangreiche Literatur über den Einfluss ökonomischer Faktoren auf das Geburtenverhalten; über die relative Bedeutung der Merkmale des Mannes bzw. der Frau und mögliche Interaktionseffekte gibt es jedoch kaum gesicherte Erkenntnisse.

Der folgende Abschnitt 2 gibt zunächst einen kurzen Überblick über den Stand der Forschung, unter besonderer Berücksichtigung schwedischer Studien. Anschließend beschreiben wir kurz unsere Daten und unser methodisches Vorgehen (Abschnitt 3), gefolgt von der Darstellung der multivariaten Ergebnisse in Abschnitt 4. Die Resultate werden in Abschnitt 5 noch einmal zusammengefasst und eingehend diskutiert.

\section{Geburtenverhalten und Erwerbsbeteiligung von Frauen und} Männern

Ausgehend von der Annahme einer effizienzsteigernden geschlechtsspezifischen Spezialisierung der Arbeitsteilung im Haushalt ${ }^{2}$, postulieren Vertreter der , Neuen Haushaltsökonomie' (vgl. Becker 1993: Kapitel 2; kritisch: Joshi 1998), dass Frauen in Partnerschaften insbesondere nach der Geburt eines Kindes ihren Erwerbsumfang

1 Siehe Andersson (1999) für eine ausführliche Darstellung der allgemeinen Geburtenentwicklung in Schweden.

${ }^{2}$ Vgl. Parsons (1949) zur Bedeutung der geschlechtsspezifischen Arbeitsteilung in Haushalt und Familie aus strukturfunktionalistischer Sicht. 
reduzieren werden, während Männer weiterhin (und möglicherweise sogar in höherem Umfang) erwerbstätig sein werden. Da die Opportunitätskosten eines Rückzugs aus dem Arbeitsmarkt positiv mit den Humankapitalinvestitionen und dem Einkommen korreliert sind, wird ein negativer Zusammenhang zwischen der Anzahl der Kinder und dem Bildungsniveau, der Erwerbsbeteiligung sowie dem Arbeitseinkommen von Frauen erwartet (Becker 1993: Kapitel 5). Weil Kinder in modernen Gesellschaften gleichzeitig einen erheblichen Kostenfaktor darstellen, wird hingegen eine positive Beziehung zwischen dem Erwerbsumfang bzw. Einkommen des Mannes und der Geburtenzahl erwartet. Tatsächlich kommen Heckman und Walker (1990) in ihrer Analyse schwedischer Daten der 1960er und 1970er Jahre zu dem Schluss, dass ein steigendes Erwerbseinkommen bei Frauen nicht nur zu einer Verzögerung von Geburten, sondern auch zu einer Reduktion der Gesamtkinderzahl führt, während für Männer der gegenteilige Zusammenhang gilt (vgl. auch Merrigan und St.-Pierre 1998). Innerhalb des selben Modellrahmens, aber unter Verwendung detaillierterer Informationen über das individuelle Erwerbseinkommen der Frau, konnte Tasiran (1995) jedoch zeigen, dass der negative Zusammenhang zwischen Fertilität und Löhnen in Schweden insgesamt geringer ausfällt, als von Heckman und Walker (1990) angenommen, und darüber hinaus nicht in gleichem Maße für alle betrachteten Paritäten und Kohorten gilt.

Obwohl also - aus einer Paarperspektive - zwei gegenläufige Einflüsse vorliegen können (deren Nettoeffekt a priori unklar ist), wird in der Literatur überwiegend allein der Zusammenhang zwischen Frauenerwerbstätigkeit und Familiengründung diskutiert (z.B. Brewster und Rindfuss 2000; kritisch hierzu: Joshi 1998; Oppenheimer 1994). Die Befunde einiger neuerer empirischer Studien unterstreichen jedoch die Notwendigkeit, die Erwerbssituation von Männern in Analysen zur Familiengründung explizit zu 
berücksichtigen. Eine wesentliche Rolle kommt hier berufsbiographischen Unsicherheiten - z.B. durch Arbeitslosigkeit - zu, die sich negativ auf die Heiratswahrscheinlichkeit sowie den Übergang zur Elternschaft auswirken können (z.B. Oppenheimer et al. 1997; Tölke und Diewald 2003). In einer Situation, in der Männer ihre Versorgerfunktion u.U. nur noch eingeschränkt erfüllen können, und in der der Anteil ihres Arbeitseinkommens am Haushaltseinkommen sinkt, wächst die Bedeutung des (relativen) Einkommens der Frau - mit einem dann möglicherweise positiven Nettoeffekt auf die Fertilitätsentwicklung (vgl. Macunovich 1996).

Eine Voraussetzung dafür, dass beide Partner zum Unterhalt der Familie beitragen können, ist jedoch die Verfügbarkeit bezahlbarer Kinderbetreuung (z.B. Stolzenberg und Waite 1984). In Schweden ist das gut ausgebaute System öffentlicher Kinderbetreuung wesentlicher Bestandteil eines wohlfahrtsstaatlichen Arrangements, das in seiner Gesamtheit auf die Förderung der Gleichberechtigung und die Vereinbarkeit von Beruf und Familie ausgerichtet ist (vgl. Andersson et al. 2004; Gustaffson und Stafford 1992). Untersuchungen des Zusammenhangs zwischen Geburtenverhalten und Einkommen bzw. Erwerbsstatus schwedischer Frauen in den 1980er und 1990er Jahren zeigen, dass in einem solchen gesellschaftlichen Kontext ein pro-zyklisches Fertilitätsmuster beobachtet werden kann (z.B. Andersson 2000; Hoem 2000), d.h. dass sich die in neoklassischen Modellen erwartete und empirisch häufig beobachtete negative Korrelation zwischen der Erwerbsbeteiligung von Frauen und Geburtenraten u.U. sogar umkehrt (siehe Ahn und Mira, 2002, für eine allgemeinere Diskussion). Vergleicht man weiterhin den Effekt der Einkommens- und Erwerbsmerkmale von Männern und Frauen auf die jeweilige Wahrscheinlichkeit eine feste Partnerschaft einzugehen bzw. ein erstes Kind $\mathrm{zu}$ bekommen, lassen sich in 
Schweden kaum geschlechtsspezifische Unterschiede feststellen (vgl. Bracher und Santow 1998; Duvander und Olsson 2001).

Bislang steht allerdings eine Untersuchung der gemeinsamen Wirkung der Einkommens- und Erwerbsposition beider Partner aus. Ohne deren gleichzeitige Berücksichtigung besteht jedoch die Gefahr, dass z.B. ein Effekt der Einkommensvariable der Frau kausal interpretiert wird, obwohl er möglicherweise nur einen tatsächlich vorliegenden Einkommenseffekt des Mannes reflektiert. Dies wäre insbesondere dann zu erwarten, wenn ein hohes Maß an Bildungs-, Erwerbs- und Einkommenshomogamie bei der Partnerwahl vorliegt (vgl. hierzu Blossfeld und Timm 1997; Henz und Sundström 2001). Die vorliegende Studie untersucht daher nicht allein die individuellen Effekte der Erwerbssituation beider Partner, sondern auch mögliche Interaktionseffekte der Merkmale von Mann und Frau. Da Paare in unserem Datensatz nur dann identifiziert werden können, wenn sie bereits ein gemeinsames Kind haben, bezieht sich die Analyse auf den Übergang zum zweiten und dritten Kind.

\section{Daten \& Methode}

Unsere Daten stammen aus den schwedischen Bevölkerungs- und Steuerregistern der Jahre 1981 bis 1999. Sie enthalten monatsgenau die vollständige Fertilitätsbiographie sowie jahresgenaue Einkommens- und Erwerbsinformationen über alle verheirateten oder unverheirateten Männer und Frauen, die mindestens ein gemeinsames Kind haben und in einem gemeinsamen Haushalt leben. Folgende primäre Einkommensarten (über die wir den Erwerbsstatus eindeutig definieren) können unterschieden werden ${ }^{3}$ :

3 Die Einkommensangaben verstehen sich vor Steuern, aber nach Abzug aller Sozialversicherungsbeiträge und inklusive Lohnersatzleistungen im Krankheitsfall oder während 
$>$ Ausbildungsförderung für Studierende: Eine Person gilt als studierend, wenn sie eine staatliche Ausbildungsförderung (als Kredit oder Stipendium) bezieht und das Erwerbseinkommen 75.000 SEK im Jahr nicht übersteigt. Praktisch alle Studierenden in Schweden erhalten eine öffentliche Ausbildungsförderung.

Arbeitslosenunterstützung oder Beihilfe während einer beruflichen Umschulung: Eine Person gilt als arbeitslos oder befindet sich in einer beruflichen Umschulungsmaßnahme, wenn sie eine entsprechende Beihilfe bezieht und das Erwerbseinkommen 75.000 SEK im Jahr nicht übersteigt.

$>$ Sozialhilfe: Eine Person gilt als Sozialhilfeempfänger(in), wenn sie Sozialhilfe bezieht und das Erwerbseinkommen 75.000 SEK im Jahr nicht übersteigt. Sozialhilfe wird Personen ohne ausreichende eigene Einkünfte oder Ansprüche auf andere Leistungen (z.B. Arbeitslosenunterstützung) gewährt.

$>$ Niedriges Erwerbseinkommen: Personen mit einem Erwerbseinkommen zwischen 37.000 und 125.000 SEK im Jahr, die zu keiner der oben genannten Gruppen gehören.

> Mittleres Erwerbseinkommen: Personen mit einem Erwerbseinkommen zwischen 125.000 und 200.000 SEK im Jahr.

$>$ Hohes Erwerbseinkommen: Personen mit einem Erwerbseinkommen zwischen 200.000 und 275.000 SEK im Jahr.

Spitzeneinkommen: Personen mit einem Erwerbseinkommen über 275.000 SEK im Jahr.

$>$ Kein Erwerbs- oder Transfereinkommen: Diese Restkategorie erfasst Personen, die zu keiner der zuvor genannten Gruppen zählen.

Knapp drei Viertel der Väter sind erwerbstätig mit einem mittleren bis hohen Einkommen, während fast vier Fünftel der Mütter lediglich über ein niedriges oder mittleres Erwerbseinkommen verfügen (siehe Tabelle 1). Vergleicht man Paare mit

der Elternzeit. Sie wurden bezogen auf das Jahr 1995 standardisiert; eine schwedische Krone (SEK) entspricht dabei etwas mehr als 10 Eurocent. 
einem Kind und solche mit zwei Kindern, zeigt sich, dass trotz der hohen Erwerbsbeteiligung von Müttern auch in Schweden ein mit der Geburt weiterer Kinder wachsendes wirtschaftliches Ungleichgewicht zwischen Männern und Frauen besteht. ${ }^{4}$

[Tabelle 1 hier einfügen]

Weitere erklärende Variablen sind das Alter des jüngsten Kindes, das Alter der Mutter, der Altersunterschied zwischen den Partnern, sowie Jahresindikatoren für den gesamten Beobachtungszeitraum. Leider enthält unser Datensatz, der aus der amtlichen Statistik stammt, kaum weitere Hintergrundinformationen über die Paare. Die abhängige Variable (Geburt eines zweiten bzw. dritten Kindes) bezieht sich auf die Geburtenordnung der Mutter. ${ }^{5}$ Im Falle einer Trennung des Paares, wenn die Mutter älter als 42 Jahre ist, oder wenn das jüngste Kind älter als 9 Jahre ist, werden die Beobachtungen zensiert. Insgesamt enthält der Datensatz rund 460.000 Zweit- und knapp 160.000 Drittgeburten. Auf dieser Grundlage schätzen wir separate ProportionalHazard-Modelle (vgl. Hoem 1987) für jede der hier betrachteten Paritäten.

\section{Ergebnisse der multivariaten Analyse}

In unserer Darstellung der Regressionsergebnisse beschränken wir uns auf die für unsere Untersuchung wesentlichen Variablen zur Erwerbstätigkeit (für weitere

\footnotetext{
${ }^{4}$ Das ist zum Großteil eine Folge der hohen Rate an Teilzeitbeschäftigten unter Frauen mit Kindern.

${ }^{5}$ Die Entscheidung, die Geburtenordnung der Mutter und nicht die des Vaters zu wählen, ist hier rein technisch begründet. Toulemon (2001) diskutiert am Beispiel französischer Daten, wann und warum sich Unterschiede zwischen den Paritäten beider Partner ergeben können.
} 
Analysen von Zweit- und Drittgeburten in Schweden vgl. Berinde 1999; Hoem und Hoem 1989; Oláh 2003). Modell 1 berücksichtigt jeweils nur die Einkommens- und Erwerbsmerkmale des Mannes, Modell 2 nur jene der Frau, und Modell 3 enthält schließlich die Informationen beider Partner.

\section{Geburt des zweiten Kindes}

In den Modellen für den Übergang zum zweiten Kind (siehe Tabelle 2) zeigt sich ein klar positiver Effekt der Erwerbstätigkeit bzw. des Erwerbseinkommens auf die Wahrscheinlichkeit einer Geburt. Im Vergleich zur Referenzgruppe - Personen mit mittlerem Einkommen - steigt sowohl für Väter (Modell 1) als auch für Mütter (Modell 2) die Wahrscheinlichkeit ein zweites Kind zu bekommen um knapp 10 Prozentpunkte, wenn sie über ein hohes Erwerbseinkommen verfügen, und gar um 20 Prozentpunkte im Falle eines Spitzeneinkommens. Die Wahrscheinlichkeit einer weiteren Geburt sinkt hingegen bei Arbeitslosigkeit, und zwar wiederum in gleichem Maße für Männer und für Frauen, nämlich jeweils um etwa 15 Prozentpunkte (vgl. auch Hank 2001; Kravdal 2002). Unterschiede zwischen den Geschlechtern zeigen sich bei Studierenden und bei Personen in Umschulungsmaßnahmen oder in Sozialhilfe, wo Frauen deutlich niedrigere Übergangsraten zum zweiten Kind aufweisen als Männer. In unserer Population - Eltern mit einem Kind - ist der Anteil dieser Personengruppen mit zusammen ca. 5 Prozent jedoch sehr gering.

Betrachtet man die Merkmale beider Partner gemeinsam (Modell 3), bestätigt sich zunächst das allgemeine Muster der nach Geschlechtern getrennt vorgenommenen Analysen. Einkommen und Erwerbstätigkeit von Mann und Frau haben dabei einen weitgehend eigenständigen Einfluss auf das Geburtenverhalten von Paaren mit einem Kind. Verfügen zum Beispiel beide Partner über ein Spitzeneinkommen, ist die 
Wahrscheinlichkeit, dass ein zweites Kind geboren wird, um ein Drittel $(1,21 * 1,10)$ höher als bei einem mittleren Einkommen. Gleichzeitig zeigt sich jedoch, dass die Übergangsrate zum zweiten Kind für Mütter mit einem mindestens hohen Erwerbseinkommen um 4 bis 8 Prozentpunkte überschätzt wird, wenn der Partner nicht in der Regression mitberücksichtigt wird.

[Tabelle 2 hier einfügen]

[Tabelle 3 hier einfügen]

\section{Geburt des dritten Kindes}

Für das dritte Kind (siehe Tabelle 3) findet sich bei erwerbstätigen Männern (Modell 1) ein U-förmiges Muster. Die Wahrscheinlichkeit einer weiteren Vaterschaft ist am höchsten in der niedrigsten Einkommenskategorie $(1,27)$, sinkt deutlich für Männer mit mittlerem Einkommen (Referenzgruppe), ist am niedrigsten bei einem hohen Erwerbseinkommen $(0,94)$, und steigt in der Gruppe der Spitzenverdiener wieder deutlich an (1,13). Die kleine - und mutmaßlich hoch selektive - Gruppe der nicht oder nur nebenerwerbstätigen Väter (Studenten, Sozialhilfeempfänger, etc.) weist unerwartet eine ebenfalls höhere, und jener von Männern mit niedrigem Erwerbseinkommen weitgehend vergleichbare Wahrscheinlichkeit für die Geburt eines dritten Kindes auf.

Bei den erwerbstätigen Frauen (Modell 2) zeigt sich hingegen ein durchgehend positiver Einkommenseffekt, der insbesondere bei den Spitzenverdienerinnen noch deutlicher ausfällt als beim zweiten Kind. Allerdings kann gleichzeitig bei arbeitslosen Müttern eine um 6 Prozentpunkte höhere Wahrscheinlichkeit für eine dritte Mutterschaft beobachtet werden als bei Frauen mit mittlerem Erwerbseinkommen (vgl. Andersson 2000; Hank 2001). Eine vergleichsweise sehr hohe Übergangsrate zum 
dritten Kind $(1,16)$ findet sich auch in der Restkategorie, die immerhin 8 Prozent der Mütter von zwei Kindern einschließt und überwiegend aus Hausfrauen bestehen dürfte, einer - zumindest in Schweden - sehr selektiven Gruppe (vgl. Berinde 1999). Lediglich die wenigen Frauen, die studieren oder sich in einer Umschulungsmaßnahme befinden, haben eine deutlich unterdurchschnittliche Neigung $(0,78$ bzw. 0,64$)$ ein weiteres Kind zu bekommen.

In Modell 3 (mit beiden Partnern) bestätigen sich wiederum die Ergebnisse der für jeden Elternteil getrennt durchgeführten Regressionen. Das U-förmige Muster bei den Männern bleibt ebenso erhalten wie die positive Korrelation zwischen dem Erwerbseinkommen und dem Übergang zum dritten Kind bei Frauen. Hieraus ergeben sich interessante Kombinationseffekte wie z.B. jener, dass Paare, in denen der Mann ein hohes und die Frau ein niedriges Erwerbseinkommen in den Haushalt einbringt, nur eine unterdurchschnittliche Übergangsrate bei Drittgeburten aufweisen $(0,94 * 0,95)$. Zwar fallen die Unterschiede zwischen den für beide Geschlechter getrennt geschätzten Modellen und dem Gesamtmodell im Allgemeinen nur gering aus; dennoch reduziert sich für Mütter mit einem Spitzeneinkommen durch die Berücksichtigung der Merkmale des Partners der Einkommenseffekt um 8 Prozentpunkte. Dies konnte bereits in der Analyse zweiter Geburten beobachtet werden und dürfte im Wesentlichen darauf zurückzuführen sein, dass in der ohnehin sehr kleinen Gruppe der Spitzenverdienerinnen (1 Prozent der Population) die Einkommenshomogamie zwischen den Partnern noch stärker ausgeprägt ist, als in der Gesamtbevölkerung (siehe Tabelle 4).

[Tabelle 4 hier einfügen] 


\section{Diskussion \& Ausblick}

Ziel dieses Artikels ist es, zur Beantwortung der Frage nach der individuellen und der gemeinsamen Bedeutung von Erwerbsmerkmalen schwedischer Männer und Frauen in Paarbeziehungen für den Prozess der Familienentwicklung beizutragen. Hierzu haben wir auf der Grundlage von Registerdaten der Jahre 1981 bis 1999 ereignisanalytische Modelle für die Geburt zweiter und dritter Kinder geschätzt. Dabei ergeben sich im Wesentlichen zwei Befunde: Erstens bestätigt sich das aus früheren Untersuchungen bereits bekannte Ergebnis, dass Erwerbstätigkeit und (mehrfache) Mutterschaft in Schweden nicht nur miteinander vereinbar, sondern sogar positiv miteinander korreliert sind (z.B. Andersson 2000). Zweitens wirken Einkommen und Erwerbstätigkeit von Mann und Frau weitgehend unabhängig voneinander auf das Fertilitätsverhalten schwedischer Paare. Die Richtung des bei beiden Geschlechtern etwa gleich starken Effektes weist auf einen grundsätzlich positiven Zusammenhang zwischen unseren Erwerbsvariablen und der Familienentwicklung hin; bei der Analyse dritter Geburten zeigt sich für Männer jedoch ein weniger eindeutiges, U-förmiges Muster.

Eine der Erklärungen für den durchgängig positiven Effekt des Erwerbseinkommens der Frau ist u.E. darin $\mathrm{zu}$ sehen, dass die öffentlichen Transferzahlungen während der ersten 12 Monate der Elternzeit (Erziehungsurlaub) an das vorherige Arbeitseinkommen gekoppelt sind. Damit stellen sie bis zu einer Obergrenze von knapp 275.000 SEK Jahreseinkommen (1999) einen echten Lohnersatz von früher 90 Prozent, heute immerhin noch 80 Prozent des vorherigen Einkommens dar. Hierdurch werden starke Anreize gesetzt, sich vor der Geburt von Kindern auf dem Arbeitsmarkt und im Beruf zu etablieren. Dies gilt insbesondere für Frauen, die auch in 
Schweden den größten Teil der insgesamt 15-monatigen Elternzeit in Anspruch nehmen (vgl. hierzu ausführlich Sundström und Duvander 2002).

Viele Paare bekommen ihr zweites Kind bereits relativ kurz nach der ersten Geburt (vgl. Andersson 1999). Kommt ein Kind innerhalb von 30 Monaten nach der Geburt des vorherigen Kindes zur Welt, beziehen sich die Transferansprüche weiter auf das letzte Erwerbseinkommen vor der Geburt des älteren Kindes, auch wenn ein Elternteil seitdem Erziehungsurlaub genommen oder die Arbeitszeit reduziert hat. ${ }^{6}$ Das Intervall zwischen dem zweiten und dem dritten Kind ist üblicherweise jedoch so groß, dass die oben beschriebenen Lohnersatzleistungen auf Basis des Einkommens nach der zweiten Geburt neu berechnet werden müssen. Dies könnte erklären, warum der von uns beobachtete Einfluss des Erwerbseinkommens der Frau auf die Übergangsrate zum dritten Kind stärker ausfällt, als beim zweiten Kind.

Hinsichtlich der Notwendigkeit, das spezifische Geburtenverhalten von Männern genauer als bislang zu untersuchen, und gleichzeitig in Fertilitätsanalysen verstärkt Merkmale von Frau und Mann zu berücksichtigen, beschränkt sich das Ergebnis unserer Untersuchung keineswegs darauf, dass eine reine ,Frauen'-Studie den Einkommenseffekt schwedischer Spitzenverdienerinnen leicht überschätzen würde. ${ }^{7}$ Die von uns beobachtete Unabhängigkeit des Einflusses der Erwerbsmerkmale beider Partner auf die Geburt weiterer Kinder ist nämlich keineswegs selbstverständlich. Sie

\footnotetext{
${ }^{6}$ Siehe Hoem (1993) für eine ausführliche Diskussion der Ursachen dieses Fertilitätsmusters mit relativ kurzen Abständen zwischen den Geburten.

${ }^{7}$ Obwohl auch dies im Kontext einer breiteren Diskussion um die Determinanten der Partnerwahl und die sozialen und demographischen Konsequenzen bildungs- oder einkommenshomogamen Heiratsverhaltens ein interessanter Befund ist (vgl. Blossfeld und Timm, 2003, für einen aktuellen Überblick).
} 
deutet u.E. vielmehr darauf hin, dass im heutigen Schweden - trotz deutlicher geschlechtsspezifischer Unterschiede etwa in der Einkommensverteilung - ein vergleichsweise hohes Maß an sozialer Gleichheit zwischen den Geschlechtern besteht, und zwar sowohl auf der gesellschaftlichen Makroebene, als auch auf der Mesoebene der Familie (vgl. Oláh 2003; Thomson und Hoem 1998).

Der schwedische Wohlfahrtsstaat hat einen institutionellen Rahmen geschaffen, der Frauen wie Männern Anreize und Möglichkeiten bietet, für ihren Lebensverlauf relevante Entscheidungen - mögen diese nun den beruflichen oder den familiären Strang ihrer Biographie betreffen - in erster Linie auf der Grundlage ihrer individuellen (materiellen) Situation zu treffen (vgl. Henz und Sundström 2001). Eine entsprechendes Maß an Autonomie ist z.B. in ,konservativen' Wohlfahrtsstaaten wie Deutschland oder Österreich kaum gegeben und dürfte für die - im Vergleich zu Skandinavien - niedrigen Geburtenraten in diesen Ländern mit verantwortlich sein (vgl. z.B. Hoem et al. 2001; Konietzka und Kreyenfeld 2003). Wie unsere Untersuchung zeigt, finden in Schweden Geburten höherer Ordnung eben nicht in traditionellen Familienkontexten, in denen der Vater die Ernährerrolle und die Mutter die Familienrolle übernimmt, statt, sondern gerade dort, wo Mütter ein hohes, und Väter möglicherweise sogar ein niedrigeres Erwerbseinkommen in den Haushalt einbringen oder eine geringere Erwerbsbeteiligung aufweisen.

Leider gibt dieser Befund noch keine befriedigende Auskunft über die konkrete Form der Arbeitsteilung im Haushalt. Leisten Männer mit einem geringeren Erwerbsumfang tatsächlich einen höheren Beitrag zur Kindererziehung und bei der Hausarbeit? Oder führt die Erwerbstätigkeit von Müttern - trotz außerhäuslicher Kinderbetreuung - häufig eher zu einer Doppelbelastung von Frauen, statt dazu 
beizutragen, die konservative Verteilung der Geschlechterrollen in Partnerschaften aufzuheben (vgl. hierzu beispielhaft die ostdeutsche Studie von Böckmann-Schewe et al. 1995)?

Es wird deutlich, dass in diesem Bereich noch ein erheblicher Forschungsbedarf besteht. Neben weiteren quantitativ-empirischen Studien (z.B. auf Basis von Daten des neuen ,Generations and Gender Survey“; vgl. Macura 2002), sollten auch die Möglichkeiten qualitativer Untersuchungen genutzt werden, um Geschlechterbeziehungen als Interaktionsprozess zwischen zwei Partnern besser zu verstehen, als dies im vorliegenden Papier allein auf der Grundlage vergleichsweise eindimensionaler Daten zum Einkommen und Erwerbsstatus möglich war. Über dieser notwendigen ,mikroskopischen' Betrachtung von Partnerschaften darf jedoch nicht vergessen werden, der sozialen und institutionellen Kontextabhängigkeit der Geschlechterverhältnisse - mit ihren Konsequenzen für die zentralen Lebensbereiche Beruf und Familie - größte Aufmerksamkeit zu schenken (vgl. Neyer 2003).

\section{Danksagung}

Wir danken dem schwedischen ,Statistiska centralbyrån“ (SCB) für die Bereitstellung der hier verwendeten Daten sowie Gerda Neyer und Angelika Tölke für hilfreiche Kommentare. 


\section{Literaturverzeichnis}

Ahn, N., \& Mira, P. (2002). A note on the changing relationship between fertility and female employment rates in developed countries. Journal of Population Economics, $15(4), 667-682$.

Andersson, G. (1999). Childbearing trends in Sweden 1961 - 1997. European Journal of Population, 15 (1), 1-24.

Andersson, G. (2000). The impact of labour-force participation on childbearing behaviour: Pro-cyclical fertility in Sweden during the 1980s and the 1990s. European Journal of Population, 16 (4), 293-333.

Andersson, G., A.-Z. Duvander \& K. Hank (2004). Do child care characteristics influence continued childbearing in Sweden? An investigation of the quantity, quality, and price dimension. Journal of European Social Policy, 14 (4), im Druck.

Baizán, P., A. Aassve \& F.C. Billari (2002). Institutional arrangements and life course outcomes: the interrelations between cohabitation, marriage and first birth in Germany and Sweden. MPIDR Working Paper WP-2002-026, Rostock.

Becker, G.S. (1993). A Treatise on the Family (Erweiterte Auflage). Cambridge: Harvard University Press.

Berinde, D. (1999). Pathways to a third child in Sweden. European Journal of Population, 15 (4), 349-378.

Böckmann-Schewe, L., C. Kulke \& A. Röhrig (1995). „Es war immer so, den goldenen Mittelweg zu finden zwischen Familie und Beruf war eigentlich das Entscheidende.“ Kontinuitäten und Veränderungen im Leben von Frauen in den neuen Bundesländern. Berliner Journal für Soziologie, 5 (2), 207-222.

Blossfeld, H.-P. \& J. Huinink (1989). Die Verbesserung der Bildungs- und Berufschancen von Frauen und ihr Einfluß auf den Prozeß der Familienbildung. Zeitschrift für Bevölkerungswissenschaft, 15 (4), 383-404.

Blossfeld, H.-P., E. Klijzing, K. Pohl \& G. Rohwer (1996). Die Modellierung paralleler und interdependenter Prozesse in der demographischen Forschung: Konzepte, 
Methoden und Anwendung auf nichteheliche Lebensgemeinschaften. Zeitschrift für Bevölkerungswissenschaft, 22 (1), 29-56.

Blossfeld, H.-P. \& A. Timm (1997). Das Bildungssystem als Heiratsmarkt. Eine Längsschnittanalyse der Wahl von Heiratspartnern im Lebenslauf. Kölner Zeitschrift für Soziologie und Sozialpsychologie, 49 (3), 440-476.

Blossfeld, H.-P. \& A. Timm (2003). Who Marries Whom? Educational Systems as Marriage Markets in Modern Societies (European Studies of Population, Bd. 12). Dordrecht: Kluwer Academic Publishers.

Bracher, M. \& G. Santow (1998). Economic independence and union formation in Sweden. Population Studies, 52 (3), 275-294.

Brewster, K. L. \& R.R. Rindfuss (2000). Fertility and women's employment in industrialized nations. Annual Review of Sociology, 26, 271-296.

Corijn, M., A.C. Liefbroer \& J. de Jong Gierveld (1996). It takes two to tango, doesn't it? The influence of couple characteristics on the timing of the birth of the first child. Journal of Marriage and the Family, 58 (1), 117-126.

Duvander, A.-Z. \& S. Olsson (2001). När har vi råd at skaffa barn? RFV analyserar 2001-8. Stockholm: Riksförsäkringsverket.

Gustafsson, S. \& F.P. Stafford (1992). Child care subsidies and labor supply in Sweden. Journal of Human Resources, 27 (1), 204-230.

Hank, K. (2001): Changes in Swedish women's individual activity status and the subsequent risk of giving birth in the 1980s and 1990s. Demographic Research [Online], 4 (4), available at http://www.demographic-research.org/Volumes/Vol4/4.

Hank, K. \& A. Tölke (2004). Männer - Das , vernachlässigte' Geschlecht in der Familienforschung. In: A. Tölke \& K. Hank (Hrsg.), Das ,vernachlässigte’ Geschlecht in der Familienforschung: Untersuchungen zu Partnerschaft und Elternschaft bei Männern (Sonderheft 4 der Zeitschrift für Familienforschung). Opladen: Leske + Budrich, in Vorbereitung. 
Heckman, J.J. \& J.R. Walker (1990). The relationship between wages and income and the timing and spacing of births: evidence from Swedish longitudinal data. Econometrica, 58 (6), 1411-1441.

Henz, U. \& M. Sundström (2001). Partner choice and women's paid work in Sweden. The role of earnings. European Sociological Review, 17 (3), 295-316.

Hoem, B. (2000). Entry into motherhood: The influence of economic factors on the rise and fall in fertility, 1986-1997. Demographic Research [Online], 2 (4), available at http://www.demographic-research.org/Volumes/Vol2/4.

Hoem, J.M. (1987). Statistical analysis of a multiplicative model and its application to the standardization of vital rates: a review. International Statistical Review, 55 (1), 119-152.

Hoem, J.M. (1993). Public policy as the fuel for fertility: Effects of a policy reform on the pace of childbearing in Sweden in the 1980s. Acta Sociologica, 36 (1), 19-31.

Hoem, J.M. \& B. Hoem (1989). The impact of women's employment on second and third births in modern Sweden. Population Studies, 43 (1), 47-67.

Hoem, J.M., A. Prskawetz \& G. Neyer (2001). Autonomy or conservative adjustment? The effect of public policies and educational attainment on third births in Austria, 1975-96. Population Studies, 55 (3), 249-261.

Joshi, H. (1998). The opportunity costs of childbearing: More than just mothers' business. Journal of Population Economics, 11 (2), 161-183.

Klein, T. (2003). Die Geburt von Kindern in paarbezogener Perspektive. Zeitschrift für Soziologie, 32 (6), im Druck.

Kohlmann, A. \& J. Kopp (1997). Verhandlungstheoretische Modellierung des Übergangs zu verschiedenen Kinderzahlen. Zeitschrift für Soziologie, 26 (4), 258274.

Konietzka, D. \& M. Kreyenfeld (2003). Neue Familienformen im konservativen Wohlfahrtsstaat? Der Zusammenhang von Eheschließung und Familiengründung in Ost- und Westdeutschland. In: J. Allmendinger (Hrsg.), Entstaatlichung und soziale 
Sicherheit - Verhandlungen des 31. Kongress der Deutschen Gesellschaft für Soziologie in Leipzig 2002 (Kongressband II). Opladen: Leske + Budrich, im Druck.

Kravdal, Ø. (2001). The high fertility of college educated women in Norway: An artefact of the separate modelling of each parity transition. Demographic Research [Online], 5 (6), available at http://www.demographic-research.org/Volumes/Vol5/6.

Kravdal, Ø. (2002). The impact of individual and aggregate unemployment on fertility in Norway. Demographic Research [Online], 6 (10), available at http://www.demographic-research.org/Volumes/Vol6/10.

Macunovich, D. (1996). Relative income and price of time: Exploring their effects on US fertility and female labor force participation. Population and Development Review, 22 (Supplement), 223-257.

Macura, M. (2002). The Generations and Gender Programme: A Study of the Dynamics of Families and Family Relationships. Mimeo.

Merrigan, P. \& Y. St. Pierre (1998). An econometric and neoclassic analysis of the timing and spacing of births in Canada from 1950 to 1990. Journal of Population Economics, 11 (1), 29-51.

Neyer, G. (2003). Gender and generations dimensions in welfare-state policies. MPIDR Working Paper WP-2003-022, Rostock.

Oláh, L. (2003). Gendering fertility: Second births in Sweden and Hungary. Population Research and Policy Review, 22 (2), 171-200.

Oppenheimer, V.K. (1994). Women's rising employment and the future of the family in industrial societies. Population and Development Review, 20 (2), 293-342.

Oppenheimer, V.K., M. Kalmijn \& N. Lim (1997). Men's career development and marriage timing during a period of rising inequality. Demography, 34 (3), 311-330.

Parsons, T. (1949). The social structure of the family. In: R. Anshen (Hrsg.), The Family: It's Function and Destiny. New York: Harper and Brothers, 73-201.

Stolzenberg, R.M. \& L.J. Waite (1984). Local labor markets, children and labor force participation of wives. Demography, 21 (2), 157-170. 
Sundström. M. \& A.-Z. Duvander (2002). Gender division of childcare and the sharing of parental leave among new parents in Sweden. European Sociological Review, 18 (4), 433-447.

Tasiran, A. (1995): Fertility Dynamics: Spacing and Timing of Births in Sweden and the United States. Amsterdam: Elsevier.

Thomson, E. (1990). Two into one: Structural models of couple behavior. In: C.W. Draper \& A.C. Marcos (Hrsg.), Family variables: Conceptualization, measurement and use. Newbury Park: Sage, 129-142.

Thomson, E. \& J.M. Hoem (1998). Couple childbearing plans and births in Sweden. Demography, 35 (3), 315-322.

Tölke, A. \& M. Diewald (2003). Berufsbiographische Unsicherheiten und der Übergang zur Elternschaft bei Männern. In: W. Bien \& J.H. Marbach (Hrsg.), Partnerschaft und Familiengründung. Ergebnisse der dritten Welle des Familiensurvey. Opladen: Leske + Budrich, 349-384.

Toulemon, L. (2001). Men's fertility and family size as compared to women's. Vortrag bei der XXIV. IUSSP General Population Conference. Salvador de Bahia, Brasilien, 18.-24. August. 


\section{Tabellenanhang}

Tabelle 1: Verteilung ausgewählter Variablen

\begin{tabular}{|c|c|c|}
\hline & $\begin{array}{c}\text { Paare mit einem Kind }{ }^{a} \\
\text { (in } \%)\end{array}$ & $\begin{array}{c}\text { Paare mit zwei Kindern } \\
\text { (in } \%)\end{array}$ \\
\hline \multicolumn{3}{|l|}{ Alter des jüngsten Kindes } \\
\hline 0 Jahre & 28 & 15 \\
\hline $1 \mathrm{Jahr}$ & 26 & 14 \\
\hline 2 Jahre & 16 & 12 \\
\hline 3 Jahre & 9 & 11 \\
\hline 4-9 Jahre & 21 & 48 \\
\hline \multirow{2}{*}{\multicolumn{3}{|c|}{$\begin{array}{l}\text { Altersunterschied zwischen den } \\
\text { Partnern }\end{array}$}} \\
\hline & & \\
\hline Mutter mehr als 6 Jahre älter & 1 & $<1$ \\
\hline Mutter 3-6 Jahre älter & 3 & 2 \\
\hline Mutter 1-3 Jahre älter & 7 & 6 \\
\hline Weniger als 1 Jahr Unterschied & 20 & 21 \\
\hline Vater 1-3 Jahre älter & 26 & 30 \\
\hline Vater 3-6 Jahre älter & 25 & 27 \\
\hline Vater mehr als 6 Jahre älter & 17 & 14 \\
\hline Erwerbsstatus des Vaters & & \\
\hline $\begin{array}{l}\text { Erwerbstätig: } \\
\text { niedriges Einkommen }\end{array}$ & 9 & 6 \\
\hline $\begin{array}{l}\text { Erwerbstätig: } \\
\text { mittleres Einkommen }\end{array}$ & 43 & 36 \\
\hline $\begin{array}{l}\text { Erwerbstätig: } \\
\text { hohes Einkommen }\end{array}$ & 29 & 35 \\
\hline
\end{tabular}

Fortsetzung nächste Seite ... 
Tabelle 1: Verteilung ausgewählter Variablen (Fortsetzung)

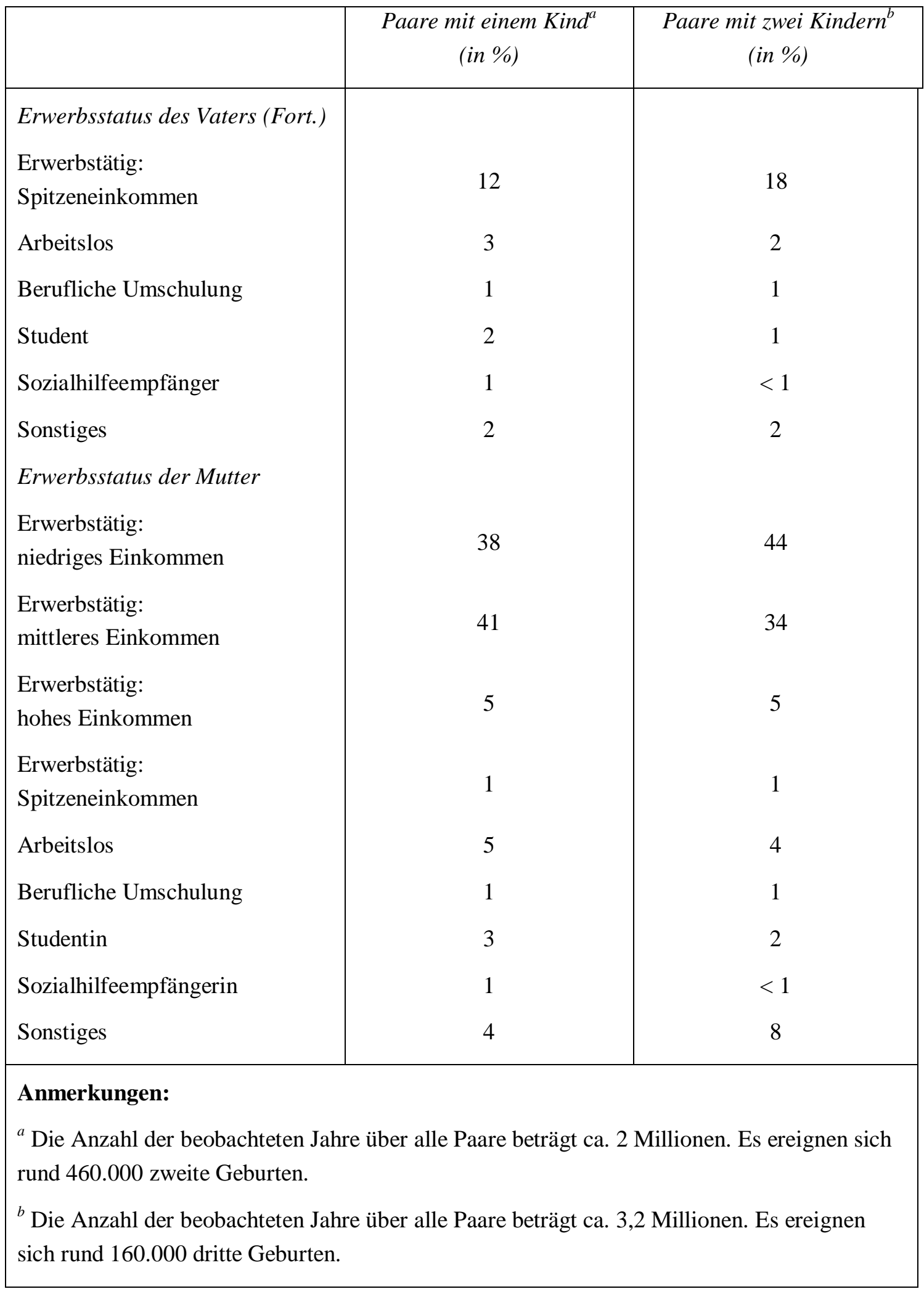


Tabelle 2: Ausgewählte Regressionsergebnisse (,relative Risiken') für Modelle des Übergangs zum zweiten Kind (Kontrollvariablen: Alter des jüngsten Kindes, Alter der Mutter, Altersunterschied zwischen den Partnern, Kalenderjahr)

\begin{tabular}{|c|c|c|c|}
\hline & $\begin{array}{c}\text { Modell } 1 \\
\text { - Männer- }\end{array}$ & $\begin{array}{c}\text { Modell } 2 \\
\text { - Frauen - }\end{array}$ & $\begin{array}{l}\text { Modell } 3 \\
\text { - Paare }\end{array}$ \\
\hline \multicolumn{4}{|l|}{ Erwerbstatus des Vaters } \\
\hline Erwerbstätig: niedriges Einkommen & 0,99 & - & 1,00 \\
\hline Erwerbstätig: mittleres Einkommen & 1 & - & 1 \\
\hline Erwerbstätig: hohes Einkommen & 1,07 & - & 1,06 \\
\hline Erwerbstätig: Spitzeneinkommen & 1,22 & - & 1,21 \\
\hline Arbeitslos & 0,83 & - & 0,85 \\
\hline Berufliche Umschulung & 0,84 & - & 0,86 \\
\hline Student & 0,94 & - & 0,99 \\
\hline Sozialhilfeempfänger & 0,70 & - & 0,76 \\
\hline Sonstiges & 0,91 & - & 0,92 \\
\hline \multicolumn{4}{|l|}{ Erwerbsstatus der Mutter } \\
\hline Erwerbstätig: niedriges Einkommen & - & 0,97 & 0,97 \\
\hline Erwerbstätig: mittleres Einkommen & - & 1 & 1 \\
\hline Erwerbstätig: hohes Einkommen & - & 1,08 & 1,04 \\
\hline Erwerbstätig: Spitzeneinkommen & - & 1,18 & 1,10 \\
\hline Arbeitslos & - & 0,86 & 0,87 \\
\hline Berufliche Umschulung & - & 0,62 & 0,63 \\
\hline Studentin & - & 0,57 & 0,57 \\
\hline Sozialhilfeempfängerin & - & 0,54 & 0,56 \\
\hline Sonstiges & - & 0,88 & 0,88 \\
\hline
\end{tabular}


Tabelle 3: Ausgewählte Regressionsergebnisse (,relative Risiken') für Modelle des Übergangs zum dritten Kind (Kontrollvariablen: Alter des jüngsten Kindes, Alter der Mutter, Altersunterschied zwischen den Partnern, Kalenderjahr)

\begin{tabular}{|c|c|c|c|}
\hline & $\begin{array}{c}\text { Modell } 1 \\
\text { - Männer- }\end{array}$ & $\begin{array}{c}\text { Modell } 2 \\
\text { - Frauen - }\end{array}$ & $\begin{array}{l}\text { Modell } 3 \\
\text { - Paare }\end{array}$ \\
\hline \multicolumn{4}{|l|}{ Erwerbsstatus des Vaters } \\
\hline Erwerbstätig: niedriges Einkommen & 1,27 & - & 1,27 \\
\hline Erwerbstätig: mittleres Einkommen & 1 & - & 1 \\
\hline Erwerbstätig: hohes Einkommen & 0,94 & - & 0,94 \\
\hline Erwerbstätig: Spitzeneinkommen & 1,13 & - & 1,12 \\
\hline Arbeitslos & 1,07 & - & 1,06 \\
\hline Berufliche Umschulung & 1,01 & - & 1,01 \\
\hline Student & 1,27 & - & 1,29 \\
\hline Sozialhilfeempfänger & 1,34 & - & 1,29 \\
\hline Sonstiges & 1,31 & - & 1,27 \\
\hline \multicolumn{4}{|l|}{ Erwerbsstatus der Mutter } \\
\hline Erwerbstätig: niedriges Einkommen & - & 0,95 & 0,95 \\
\hline Erwerbstätig: mittleres Einkommen & - & 1 & 1 \\
\hline Erwerbstätig: hohes Einkommen & - & 1,13 & 1,11 \\
\hline Erwerbstätig: Spitzeneinkommen & - & 1,47 & 1,39 \\
\hline Arbeitslos & - & 1,06 & 1,05 \\
\hline Berufliche Umschulung & - & 0,78 & 0,76 \\
\hline Studentin & - & 0,64 & 0,62 \\
\hline Sozialhilfeempfängerin & - & 1,05 & 0,93 \\
\hline Sonstiges & - & 1,16 & 1,14 \\
\hline
\end{tabular}


Tabelle 4: Individuelles Erwerbseinkommen und Erwerbseinkommen des Partners (zusammengefasste Registerdaten der Jahre 1981 bis 1999, eigene Berechnungen)

\begin{tabular}{|l|c|c|c|c|}
\hline & \multicolumn{2}{|l|}{$\begin{array}{l}\text { Anteil der Frauen (in \%) mit ... } \\
\text { Erwerbseinkommen \& } \\
\text { Partner in gleicher Kategorie }\end{array}$} & \multicolumn{2}{l|}{$\begin{array}{l}\text { Anteil der Männer (in \%) mit ... } \\
\text { Erwerbseinkommen \& } \\
\text { Partnerin in gleicher Kategorie }\end{array}$} \\
\hline & mit 1 Kind & mit 2 Kindern & mit 1 Kind & mit 2 Kindern \\
\hline$\ldots$ niedrigem ... & 10 & 7 & 42 & 49 \\
$\ldots$ mittlerem ... & 43 & 33 & 42 & 32 \\
$\ldots$ hohem ... & 36 & 37 & 7 & 5 \\
$\ldots$ Spitzen- ... & 56 & 62 & 7 & 5 \\
\hline
\end{tabular}

